\title{
Evaluation of Maternal Death Surveillance and Response System in Dewachefa Woreda, Oromia Zone, Amhara Region, Ethiopia, 2018
}

Mengistie Tariku ( $\sim$ mengesh2009@gmail.com )

\section{Research article}

Keywords: Dewachefa district, Maternal death surveillance and response, Surveillance evaluation

Posted Date: May 26th, 2020

DOI: https://doi.org/10.21203/rs.3.rs-27912/v1

License: (c) (i) This work is licensed under a Creative Commons Attribution 4.0 International License.

Read Full License 


\section{Abstract \\ Background}

Maternal death surveillance and response (MDSR) is the "litmus test" of the health system that provides evidence for accomplishment, links activities to results, makes maternal death visible at all levels, informs communities \& health workers, increases country ownership of data, provides information in real time and allows improvement towards catching all maternal mortalities. The aim of study was to evaluate maternal death surveillance and response system in Dewachefa.

\section{Methods}

A cross sectional study design was conducted in two health centers, five health post, district health office and from these facilities 32 health workers were included. Data were collected through focal person, health worker and health extension worker interview by using checklist. Collected data were entered into Epi data version 3.1. These data were exported to statistical package for social science for analysis. Analyzed data were presented in the form of text, table and figures.

\section{Result}

The average completeness of weekly report form of the district was $77.4 \%$. Twenty-eight (87.5\%) of the health worker had not got Maternal death surveillance and response (MDSR) training. All visited health facilities and Woredas focal person were trained. The system had under notification of maternal death from the community, poor involvement of health facility staff, and discordance of data between public health emergency management, and maternal and newborn health unit report. Establish rapid response team that includes maternal and child health staff's maternal death review committees in all health facilities.

\section{Background}

World health organization (WHO), Federal ministry of health maternal, newborn and child health (MNCH) unit, and Public Health Emergency Management (PHEM) unit within Ethiopian public health institute, regional health bureau, zonal health department, District health office, health facilities and any community are the stakeholders of maternal death surveillance and response (MDSR) system [1].

Globally, an estimated 287000 women died from pregnancy and its complications in $2010,99 \%$, of them were in developing countries [2].

In Ethiopia, about 13,000 women died from pregnancy related conditions in 2013 , making the country maternal death ratio $420 / 100,000$ live births and providing approximately $4 \%$ to the total maternal deaths 
[3].

Even where resources were limited, almost all maternal deaths are preventable and could be eliminated. Surveillance system is a vital component of elimination approach that not only ways the records of deaths but delivers signal about the main factors contributing to maternal deaths - and how these deaths could be managed [1].

In 2012, the World Health Organization (WHO) and partners introduced the Maternal Death Surveillance and Response (MDSR) approach as a new method to maternal death review [4]. Maternal death is often defined as the "litmus test" of the health system that measures a program's ability to answer to women's health requirements, mainly throughout and after pregnancy and birth [5].

Most countries with high maternal death have weak public registration systems. Therefore, numerous maternal deaths and the explanations overdue these deaths remain unregistered and unreported, mainly when women die at home [6].

Factors that enable to establish MDSR is Political will at regional health bureau to impulse through change well established Health Development Army structures ability to take concern for action rather than waiting for community level variation [7].

The aims of MDSR are to deliver information that guides immediate as well as longer term actions to reduce maternal death; and to count every maternal death, allowing an assessment of the true magnitude of maternal death and the influence of actions to decrease it $[2,3]$.

MDSR is an uninterrupted system that shapes on recognized methods. Maternal death review (MDR is one of the systems that has directed to local strategy variations and improvements in the quality of maternal health services, even in challenging situations. MDSR builds on the effort is implemented on MDR and helps to understand the events surrounding maternal deaths. It focuses on the need to respond to each maternal death with actions to prevent similar deaths in the future, and to collect data on all maternal deaths using clearly defined data sources and processes for identification and notification [2, 3, and 4].

MDSR also provides evidence for accomplishment, links activities to results, makes maternal death visible at all levels, informs communities \& health workers, increases country ownership of data, provides information in real time and allows improvement towards catching all maternal mortalities [7].

If accurate information is available, preventing maternal deaths can be achievable to provide targeted actions. MDSR makes each maternal death a notifiable event, and confirms that communities and facilities report and respond to end preventable maternal deaths [6]. The aim of this study was to evaluate maternal death surveillance and response system in Dewachefa.

\section{Methods}




\section{Study setting and period}

Cross sectional study design was conducted from March 1-30/ 2018 in Dewachefa district, North- East of Ethiopia. The district is located about 325 Kilo meters from Addis Ababa (the capital city of Ethiopia) and 555 Kilo meters from Bahir Dar (the capital city of Amhara region) at an altitude of 1623 meter to 2570 meter above sea level. The district is bordered by Majetie district in the South, South Wello in the West, Dewi Harewa in the Northwest, Artuma fursi district in the Northeast and the East. The area of the district is 782.22 square kilometer with the total population 151645 . The district is governmentally alienated into 26 kebeles. There are7 health centers and 26 health posts in the district [8].

\section{Sample size determination and sampling technique}

Dewachefa district was purposively selected based on the criteria whether the surveillance evaluation was conducted before and the number of maternal death report during the previous nine months. Amongst the total health centers in the district, $2(25 \%)$ of the district health centers were included in the study. These health centers were carefully chosen by lottery method simple random sampling. From these health centers; all health posts were involved in this evaluation. Equally, health professionals in the health centers and health extension workers in the health posts were invited to be included in the study.

\section{Data collection procedures}

Structured self-administered technique was used to collect data from health workers and health extension workers to assess their knowledge and attitude towards the surveillance attributes and operations of the MDSR system [1, 4]. An interviewer guide was used to collect data from district PHEM and $\mathrm{MNCH}$ officers, health centers PHEM focal persons and health extension workers. A checklist, using MDSR guidelines on surveillance system evaluation was used to gather data on the system attributes [1]. MDSR identification, notification and weekly and case-based report formats were reviewed to check for data quality, completeness and timeliness of the system.

\section{Data analysis}

Epidata versions 3.1 was used to enter collected data. Entered data were exported into statistical package for social science (SPSS) version 20 for data clearness and analysis. The findings of this evaluation were stated in the form of text and tables.

\section{Result}

\section{Socio demographic characteristic of participant health workers}

Thirty-two health workers were participating during the evaluation. Seventeen (53.1\%) of them were male. The median age of participants was 28.5 years. Regarding to professional background, twelve (37.5\%) of 
the total were nurses. The mean year of experience of health worker was 8 years.

\section{Knowledge of health workers on MDSR}

Twenty-eight (87.5\%) of the health worker had not got MDSR training. Among the total health workers, nineteen (59.4\%) were not define the case definition of maternal death. Fifteen (53.6\%) of heard about MDSR were not have good knowledge about MDSR (Table 1).

Table 1: knowledge of participated health workers on MDSR in Dewachefa, Oromia zone, Amhara region, Ethiopia, 2018

\begin{tabular}{llcc}
\hline & Variable & Frequency & Percentage \\
Training on MDSR(n=32) & No & 28 & 87.5 \\
& Yes & 4 & 12.5 \\
Heard about MDSR(n=32) & Yes & 28 & 87.5 \\
& No & 4 & 14.3 \\
Heard from(n=28) & Training & 4 & 24.3 \\
& Orientation & 17 & 60.7 \\
& Reading & 3 & 10.7 \\
Participating in MDSR & Others & 4 & 14.3 \\
& Yes & 14 & 43.8 \\
& No & 18 & 56.3 \\
Mefine maternal case definition & Yes & 13 & 40.6 \\
& No & 19 & 59.4
\end{tabular}

\section{Surveillance attributes}

\section{Simplicity}

Eighteen (56.3\%) of health workers had positive attitude toward simplicity of the surveillance system. All health centers surveillance focal person and woreda PHEM focal person agree the simplicity of the system (Table 2). 
Twenty (62.5\%) of health workers believes that system adopts to the user improvement demands (Table 2).

\section{Acceptability}

Twenty (62.5\%) of health workers had positive attitude toward the acceptability of the system.

Dewachefa woreda accepted the notifiability of maternal death. Four health posts (80\%) of the total visited health posts were not reported suspected maternal deaths. Verbal autopsy was not done by all visited health posts. All visited health centers focal persons accepted the system. All visited Health facility and woreda health office sent weekly report to the next level by hard copy. Reported data were not analyzed at woreda and visited health facilities. Rapid response team was not functional at woreda and visited health facilities. Action for response was not developed in all visited health facilities and woreda health office (Table 2).

\section{Predictive positive value}

Twenty-one (65.6\%) of health workers believes that death reported in this system are actually maternal deaths. Positive predictive value was not calculated because 12 suspected maternal death were not identified and notified by health centers (Table 2).

\section{Sensitivity}

Among the total participant health workers, $23(71.9 \%)$ of health worker had positive attitude toward the sensitivity of the system. Maternal death reported to the woreda MNCH unit were 5 in 2017/2018. Maternal deaths notified to Woreda PHEM unit were 3 in 2010. The sensitivity of this surveillance was 3/5(60\%) (Table 2).

\section{Representativeness}

Twenty-one (65.6\%) of health workers perceived that maternal death report represents the situation in the facility/community. Twelve suspected maternal death were not identified and notified from the community in 2017/2018 of the first nine month. The representativeness of the system in MNCH unit was 5/17(29.4\%). In PHEM unit its representativeness was 3/17 17.6\% (Table 2).

\section{Data quality}

\section{Timeliness}

Twenty-three $(71.9 \%)$ of the health workers agree with MDSR data is always ready when we need it for planning purposes. All maternal death report of 2017/2018 was notified after 8 day of death. The casebased report forms of 4 maternal deaths were sent to the woreda after 1 month of death. One case-based report format was reported from health facility to woreda after 3 months (Table 2).

\section{Completeness}


The revised weekly reports were 31 for all visited health facilities and woreda health office. Among the total revised weekly report form, the report forms left black were $7(22.6 \%)$. The report completeness of the woreda was $24 / 31$ (77.4\%) (Table 2).

\section{Cost}

Nineteen $(59.4 \%)$ of health workers agree with the system is not costly as compared to the current benefits we gain from it. The mean annual financial cost of the system could be 55859 Ethiopian birrs in each health facilities and woreda health office (Table 2).

\section{Usefulness}

Nineteen (59.4\%) of health workers said current system have an ability to estimate maternal death in the facility/community, show the trend of maternal death in the facility/community, the progress and effect of preventive and control methods applied against maternal death and indicate major causes of maternal deaths in the health facility/community (Table 2).

Table 2: Attitude of participated health workers toward the attribute of surveillance system in Dewachefa, Oromia zone, Amhara Region, Ethiopia,

\begin{tabular}{|c|c|c|c|}
\hline \multicolumn{2}{|l|}{ Variable } & Frequency & Percentage \\
\hline \multirow[t]{2}{*}{ Simplicity } & Positive & 18 & 56.3 \\
\hline & Negative & 14 & 43.8 \\
\hline \multirow[t]{2}{*}{ Flexibility } & Positive & 12 & 37.5 \\
\hline & Negative & 20 & 62.5 \\
\hline \multirow[t]{2}{*}{ Acceptability } & Positive & 20 & 62.5 \\
\hline & Negative & 12 & 37.5 \\
\hline \multirow[t]{2}{*}{ Sensitivity } & Positive & 23 & 71.9 \\
\hline & Negative & 9 & 28.1 \\
\hline \multirow[t]{2}{*}{ Predictive value positive } & Positive & 21 & 65.6 \\
\hline & Negative & 11 & 34.4 \\
\hline \multirow[t]{2}{*}{ Representativeness } & Positive & 21 & 65.6 \\
\hline & Negative & 11 & 34.4 \\
\hline \multirow[t]{2}{*}{ Timeliness } & Positive & 23 & 71.9 \\
\hline & Negative & 9 & 28.1 \\
\hline \multirow[t]{2}{*}{ Cost } & Positive & 13 & 40.6 \\
\hline & Negative & 19 & 59.4 \\
\hline \multirow[t]{2}{*}{ Usefulness } & Positive & 11 & 34.4 \\
\hline & Negative & 21 & 65.6 \\
\hline
\end{tabular}

\section{Stability}


Woreda health office PHEM, MNCH units, and health center PHEM and MNCH focal had trained focal person (Table 1).

All visited health posts were not using revised PHEM tools for maternal death surveillance and response whereas all visited health centers and woreda health office were using revised PHEM tools.

\section{Discussion}

The average completeness of weekly report form of the woreda was $77.4 \%$. This is consistent with the study conducted Zimbabwe 79\% [9] and in Mutare district, Zimbabwe76\% [10].

The timeliness of this surveillance system was poor because all reported maternal death report sent from health facility to woreda office after 8 days later. The average time of case-based form notified from health facilities was 2 months which is greatly discrepancy the standard FMOH report schedule 48 hours. This might be due to fear and blame. The management looks at it from a negative point of view, that they will be held responsible for the maternal deaths [7].

Maternal death surveillance and response system was not representative because 12 suspected maternal death reported did not reported from health post/ community. This is greatly contradicted with the FMOH MDSR which recommends all suspected maternal death report from community/health post [1]. This might be due to weak supervision and feedback system.

The sensitivity of this surveillance system was $17.6 \%$. This is discrepancy with the study conducted in Cameron $42.9 \%$ [11]. This might be due to unavailability of maternal identification and notification form at the health posts, absence of feedback and supervision system.

This system is unstable because all visited health posts were not using revised PHEM tools for maternal death surveillance and response, and there was not established feedback and regular supervision system. This is contradicted with the FMOH target which recommends all facilities have revised PHEM tools [1]. This might due to week coordination between woreda PHEM and MNCH units.

Woreda health office PHEM and MNCH unit had delegated officers. The visited health centers had PHEM and $\mathrm{MNCH}$ focal persons. This is consistent with the target of $\mathrm{FMOH} 100 \%$ [1].

The proportion of woreda PHEM and $\mathrm{MNCH}$ unit officer, health center focal person training was $100 \%$. This is consistent with the target of $\mathrm{FMOH} \mathrm{100 \%} \mathrm{[1].}$

\section{Conclusion}

The system had under notification of maternal death from the community, poor involvement of health facility staff, and discordance of data between PHEM and MNCH report. The system was not representative, less sensitive, poor quality of data, less useful and unstable. General, the System is not sustainable/ not standardized. 
Establish RRT that includes maternal and child health staff's maternal death review committees in all health facilities

\section{Limitation}

This study cannot measure the impact of MDSR system because there are not baseline data about maternal death in all visited health facility and woreda health office.

\section{List Of Abbreviations}

CDC: Center of disease control, FMOH: Federal ministry of health, MDR: Maternal death report, MDRF: Maternal death report form, MDSR: Maternal death surveillance and response, MNCH: Maternal, newborn and child health, PHEM: Public health emergency management, RRT: Rapid response team, VA: Verbal autopsy, WHO World health organization

\section{Declarations}

\section{- Ethics approval and consent to participate}

Ethical clearance was obtained from Oromia Zone Health department Ethical review committee. Written permission was obtained from district, Health centers and Health posts administrative bodies.

- Consent for publications: Not applicable

- Availability of data and material: The data sets generated during the current study are available from corresponding author on reasonable request.

- Competing interest: The authors declare that they have no competing interests

- Funding: Not applicable

Author's contributions

MK wrote study design, data entry, analyzed the data, and drafted paper.

Author read and approved the final manuscript

\section{Acknowledgments}

I would like to acknowledge Bahir Dar University, College of Medicine Health Sciences and Department of Epidemiology and Biostatistics.

My sincere and deepest gratitude would go to Ethiopian Public health Institute, Oromia Zone Health department and Dewachefa woreda health office. I would also like to extend my thanks to data collectors, 
supervisors and study participants.

\section{References}

1. Amha, Kebede, PHEM implementation manual for MDSR, edition 1, 2016.

2. WORLD Health Organization. MDSR Technical guidance: Information for Action to Prevent Maternal Death. In. Edited by WHO. Geneva. Switzerland: WHO Document Production Services; 2013.

3. WHO. Trends in maternal mortality: 1990 to 2013. Estimates by WHO, UNICEF, UNFPA, the World Bank and the United Nations Population Division. In.; 2014.

4. World Health Organization (WHO). Maternal Death Surveillance and Response: Technical Guidance. Information for Action to Prevent Maternal Death. Geneva: WHO; 2013.http://www.who.int/maternalchild_adolescent/documents/maternal_death_surveillance/en/ Accessed March 1/ 2018.

5. World Health Organization (WHO). Beyond the Numbers: Reviewing Maternal Deaths and Complications to Make Pregnancy Safer. Geneva: WHO; 2004.http://www.who.int/maternalchild_adolescent/documents/9241591838/en/ Accessed March 2, 2018.

6. Matthews Mathai TR, Dilip. Issrah Jawad, Maternal Health Strengthening accountability to end preventable maternal deaths. International Journal of Gynecology Obstetrics. 2015;131:53-5.

7. Professor Wendy J Graham and Dr Ephrem Tekle Lemango Strengthening the health system in Ethiopia through maternal death surveillance and response Mexico 18-21 October 2015.

8. Dewachefa 2018 fiscal year annual plan, Kemise, July 2018.

9. Rutgers S. Two years maternal mortality in Matabeleland north Province, Zimbabwe. Cent Afr J Med. 2001 Feb;47(2):39-43.

10. Mafaune FMutsigiri-Murewanhema,PTrish. Tsitsi Juru, \& Notion Tafara Gombe, Donewell Bangure, Mufuta Tshimanga Evaluation of the maternal mortality surveillance system in Mutare district, Zimbabwe, 2014-2015: a cross sectional study. Pan African Medical Journal. 2017;27:204. doi:10.11604/pamj.2017.27.204.7210.

11. Gael Kouamen. Evaluation of the maternal death surveillance and response (MDSR) system of the Northwest and center regions of Cameroon:2016. 